MVFS-THGIRVVYNTAN----VDRSLYAVVHCSDGKYAEVSKDHLNRLKFIEKSSEEEDI 55 TC MASF-TSCARILYNTTN----VDRTMHTIVHCSDGKYVEVPKAVADKCPFIEKVKEEMIP 55 Lm MADEGAIDIRLVLKGPNGEFKVDRDLYIIVHCDDGKYIEVSKNYIKQCPFIEEA-EGEIP 59

EFDYPEAVLESIAQWTLKYGMDGVAVSSTVRPCIYRNVQHVLKDDWEREFFTTRLMNDLN 115 EFEYPAAVLENLVCWTEHYGIDGFAESEIVRPCIYRNILHVLKNKWDNGFFVSRLTSELN 115 EFGYPAAVLEHLIRWAVHYGVDGHAASOLTRPCIYRDFSYVVTDKWDNDFFNQRLCSPLN 119

IMHYLQT INAAEKYEMKGFHDFLCVCLSCKLRNEEDTELVHKIMGLEDFVEIGPSDLDEA 175 IGHYLQTVNAAEKFNMKGLHCFLCVGLSCKFRSEDDAELIHKIMGLPSNVQPNQEDIENV 175

B --MSYRAYG----GGYRGRGGRGGRGGQHWQQTACEEDTF-RDPSN-----FNSGSP--- 45
--MYHRGYG----GHYRGGRG-GGRGHSHWHNNAPMERGG-HMPSNNEGAAISSGSTG-- 50
MSSYYRGGSTRGRGHYRGGGEGGFRRGRGSYADAVSDGNMNRNEEQQRGERYRSQSSGDD 60

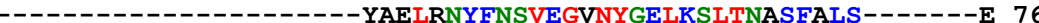
------------------FSPLMDFFHSVEGRNYGELRSLTNETYQIS-------E 81 ESERGSGGFGGNPQCHAGPPLPLVALESFLRDVDGSNYSQLKQLTGRTYSLTRQMKPDPA 120

SVRCTFLAIQSDPFAPGSQVRMSCPCPFQLQKILQCSELSS--------------CVPC 121 NVRCTFLSIQSDPFAPGSQVRLVCPCTFSLEKVLQTTDLAA-------------ANPC 126 SVSIRFVRIQPDPFAPGSQVCVSVPAPFATHALLHSAHSTPPGVTAAALGNLLSPEDVAC 180

RRIAAEDYILRSFHDGHRRGVARHHSGALHVMRPSQHVLERSTVVLVNG---DRERGG-- 176 RRVAAEDFILRSFHAGYRNGI PRRTSGAVOVLRPSOHVLERSTVGLVKA---HOOKSGMO 183 RRVAAEDFILRCVKGGFAAG--RNTSGAVQTIHQSQHVIARSAVRLLDASEAHDSDGTSD 238

VEVHLFARVKLPGHGRRIDGHGAIRI FYDELLPIMERCVVGLDEEALYQHVTCVHDQEHL 236 AE IE I FARVKL PGHGRR ID GHGA IDI FYNELVPLLEQCVVGLNEEDLHQHVICVHDQEVL 243 GWIHVYFRVKLPGHGRRIDGRRIQQILFSEVLPVFQENVLRCDHHALWAHVTCVHDQEWL 298

\title{
A-loop
}

RGELRAAGCVAFVANGSVLPRESGNSDRPLG-KGAVPFVSPASLERTFHLPHSGATVTGM 295 RSNLLGAGYVAFVANGAILPRDAGNSDKPLR-DNAVPFQSPKSLECSFTLPHSGKTITGM 302 RAQLREWGFAAFIADGAILPRATGNSDKPLSGPSVVAFSPPDTLRHTFQLPYSGRAISGA 358

\section{Walker A}

GLPHGLILIAGGGFHGKSTLLRALEVGIYNHVPDDGRVYVTVDPTAVKIRAEDRRSINGV 355 GLPPGLTLIAGGGFHGKSTLLRALEVGIYNHVPDDGRTYVVVDPTAVKIRAEDRRSVHGV 362 GLPHGLTLIAGGGFHGKSTLLRALELGVYNHVPDDGRTFVVVDPTAVKIRAEDRRAVHGT 418

$$
Q \text { loop motif } C
$$

Walker B D loop

DISPFINNLPFQKTTTSFVTSDASGSTSQAANIMEALELGSRLILLDEDTCATNLMYRDD 415 DISPF INNLPFGKTTNFFVTADASGSTSQAANIMEALELGSQLLLLDEDTCATNLMYRDA 422 DISPFITNLPYRDNTTAFVTADASGSTSQAANIMEALELGSTTLLLDEDTSATNFMYRDP 478

\section{H}

LMQQLVPREQEPITPFVDRVTDLIQNHGVSSIMVIGGSGQYFPHATVTLVMNAYKAFDCS 475 LMOMLVPRAQEPITPFVERVADLSQNHGVSSIMVIGGSGQYFPQARVVLVMNAYQISDCT 482 LMEQLVPRTQEPITCFVHRVRDLMYHHGVSVIMVVGGSGQYFPMADVVLLLNAYKVTDAT 538

ERAKEISR-------TFMSSVQPPKKAISAFVSPMQRQFDGSGTFSTVHCRRGHD---- 523 KEAKE IASN-------SSLPALNP PGDTASVFI PDVNRCFDPDGSFTTVRRRRGRE---- 531 AQAKAIAAQSVGRGGVGLVESAAPP--ATSAFRLPPRRSFVYADTFGRLGSQSQQQQYHH 596

--------SIKVSGVGVDSIRFAEETIDLSLVEQIVEEGQVNAIAQCLAMLYDGEEN 572 ---------GTKVSGIGTESIRFSEETIDLSMVEQIVEEGQVNAIAQCLALLYDGEPR 580 HHGYGYGGSGRGIKISGSGIEHIRIADEDINVTLLEQLVEEGQLNAIAQCLAMLYDSGSA 656

GIRTILAKGK-------SLKLLYSPSGGSEP-RNVPFYSEFAALIEGCDAALRDARLEAR 624 IVPEMTTKGG------ALTQLPS PGGVCEI-QRGKFNSNFSSMIAGCCSHOHDKRLELR 632 AAAEWQREEAPRSRYPPSLSKALSVDAAAAAGAPASSLSDFGRLVYNCEGRLRQARLELQ 716 
C

TPSCYLPRGFTSAARRFEIGAALNRIRTLRTLTAAMK 661 TPSCYLPRGFTSATRHIEIGAALNRLRTLRTVTAKR- 668 TASCYLPVGFTALPRVFEIGAALNRLRTLVTSCK--- 750

SAM 2

MS--SAVDSLLVRLKI PDLI PKFREMNVDRIISLRKLSEEELRTAVPDDDORNRIIEAIK 58 MN--SAVDSLLMRLKLSDLI PKFKEMKLDRIVNLRKLSEEELREAVPDDEQRNLIIHAIS 58 MSQQNAVEALLARLQMGDTIPAFQSMGVDRIVSLRKMSEEALSQAVPDPEQRQALMDAIE 60

NRGSSE-KRTQAPAASINPPRSTD-DGGRGGMSYPRG--APRGGGPVRGRGRGGTGSAGE 114 NRGTHE-KRQQSQAANINPPRNAD-DGGRVGMSFPRG--STRGG-MGRGVSRGGRGGGST 113 SRGHLQSRRAGPPAGQPHPPRSADADFARGGMDEGRGGYAPRGD-RGGRIARGGRGGG-- 117 ZF

GGFHGSGSNNANMFPSRORVCNHFLNGD-CRYGDSCRYSHN----KALCO-EAAESVPRR 168 N--NNSNSETNTSFPGRQRACNHFFSGE-CKFGDRCRYSHD----KEIYDREMAENGPRR 166 ---RGAGAPNG---DGAARVCRHVFTPEGCKYGDKCRYSHDENDRRRAQEVPTGSGMAQR 171 $\mathrm{KH}$

NHDATSLSEDFSETCI I PTHRIKYLLANRADRLRS I HSKNRTHNKSFOHIDPTIEKFELV 228 PNDSVSKPKDYSEVCEIPTHRIKFLLAKKAERLKQIHIQNHTHNEPFKRVDSNVEKFELV 226 SLERPPPNFKFSIEVEIPTERIKYLLGSQGNNMKF INETCGTYNERFDHVDEKEETFTIR 231

IYGADQQSVQESKKMILACIGVTREEEQKNRVQYTMNELSSNQRAAKFLAACNMKNEGTV 288 VYGPDPQSVLQSKKMILSYVGVTKQEEQKSRLQYTINELSSNQHAAKLLAACNIKNEGTA 286 ILGGSEEAINKAKEMLLLSAGVKREEEKKDRFQYAVNELDANTHAARLFAACNTKNKDTT 291

RELSEASLRNIISFF'RFEKQQDIRHFWVNTN-SEHSKLDIIANIVAQLQGVQAIMFCDQK 347 RELSEETLKSIISFFRFEKQODVRHFYLNVN-HDRVKLDKVAKIVAQLRGVQAIMFSDQK 345 RHLSDSIMRNIVSSFHFVRPQEVRHFYMYTSSSDKDKLEMVSKIVSQLKGVQAILFCDQK 351 Helicase

RVVDMSKVASKITRYFNGVSPLFLHRAIPKEERMKMLOTFKDGKPNENGIRERLIVTNED 407 RVMEMSKVASKIARFFNGVTPLFLHREIPKEERMRILQEFKDGEENESGIRERLLITNED 405 RVEEMCKGSQRIARHFNGVEPQFVYRQLSKEDRMMALERFKKGVENENGVRQRLLVTNED 411

YAKLARKTIVPYVNLVINYSVPRSEEYYLLQSLVAGRSDTVGVSIVCVFPHEQSLFQELQ 467 YAKLARKTLIPYVNLVINYVVPRTEEYYLLQSLVAGRSDTVGVSILCVSPYDQSTFHELQ 465 YAKLARKTII PYVNLVINFSVPRAEEYYVLQSQVAGRQGTIGASFLCVSNYEEALFRELE 471

S2 Fig. Sequence alignments were performed using ClustalW multiple sequence alignment program (http://www.genome.jp/tools/clustalw/). The alignment with the trypanosomatid species; Trypanosoma brucei (Tb), Trypanosoma cruzi $(\mathrm{Tc})$, and Leishmanina major (Lm), are presented. Residues shown in red, blue and green represent identity, similarity and weak similarity, respectively. The sequences were obtained from GeneDB. (A) Sequence alignment of T. brucei p22 (Tb927.7.7460) with its homologues. The PDIb (protein disulfide isomerase) is indicated. (B) Sequence alignment of T. brucei p72 ATPase (Tb927.3.1590) with its homologues. (C) Sequence alignment of T. brucei ZC3H41 (Tb927.11.1980) with its homologues. The different domains are indicated; SAM, sterile alpha motif; ZF, zinc finger; KH, K homology domain. 